\title{
3D MODELING FOR WILDLIFE ENCYCLOPEDIA USING BLENDER
}

Sallar Khan

Sir Syed University of Engineering and Technology. Karachi, Pakistan. E-mail: sallarkhan_92@yahoo.com

Sallar Ghanna Sir Syed University of Engineering and Technology. Karachi, Pakistan.

E-mail: salarchana55@gmail.com

Syed Abbas Ali

N.E.D University of Engineering and Technology. Karachi, Pakistan.

E-mail: saaj.research@yahoo.com

Muhammad Haaris Khan

Sir Syed University of Engineering and Technology. Karachi, Pakistan.

E-mail: haariskhan255@gmail.com

Arhum Hayat Qazi

Sir Syed University of Engineering and Technology. Karachi, Pakistan.

E-mail: arhum.hayat@gmail.com

Kamran Mengal

Sir Syed University of Engineering and Technology. Karachi, Pakistan. E-mail: kami_mengal@hotmail.com

Recepción: 30/07/2019 Aceptación: 20/09/2019 Publicación: 06/11/2019

\section{Gitación sugerida:}

Khan, S., Channa, S., Ali, S.A., Khan, M.H., Qazi, A.H. y Mengal, K. (2019). 3D modeling for Wildlife Encyclopedia using Blender. 3C Tecnología. Glosas de innovación aplicadas a la pyme. Edición Especial, Noviembre 2019, 133-147. doi: http://dx.doi. org/10.17993/3ctecno.2019.specialissue3.133-147

\section{Suggested citation:}

Khan, S., Channa, S., Ali, S.A., Khan, M.H., Qazi, A.H. \& Mengal, K. (2019). 3D modeling for Wildlife Encyclopedia using Blender. 3C Tecnología. Glosas de innovación aplicadas a la pyme. Speciaal Issue, November 2019, 133-147. doi: http://dx.doi. org/10.17993/3ctecno.2019.specialissue3.133-147 


\section{ABSTRACT}

In this modern era of technology, the ways of learning are changing drastically, and $3 \mathrm{D}$ is becoming one of the popular learning methods around the globe for experts and researchers. While it has been observed from past studies that the wildlife researchers, students, and experts need different sources like books and websites to gather and learn information about wildlife, which may be a time taking task and old approach seems to be. In this paper, we have used a 3D creation software blender for developing live 3D models of different species and providing a complete user friendly control to the user which will assist them to retrieve any information about different species from region to region around the world. These models are less complex and more attractive way of learning for the user.

\section{KEYWORDS}

3D Modeling, Blender, Wildlife, Animation. 


\section{INTRODUCTION}

The universe of 3D illustrations is an intricate field, powering ventures from gaming and film to design and building. 3D specialists and fashioners utilize explicit procedures and procedures like 3D visual computerization, rendering, perception, and activity to breathe life into a dream onscreen. Be that as it may, we are in the beginning of another kind of vivid registering, where 3D models will turn into a run of the mill resource we will almost certainly find on sites. The utilization of 3D introductions, as fundamental method for showing items or administrations in another period of web innovation wherein the online world, as observed from our perspective, there is a wide scope of choices for the acknowledgment of 3D plan where it very well may be utilized in a one of a kind, and unique way, showing the article from various perspectives, from the presentation of the subject's detail to connection or mobility of parts. 3D models can speak to any item, any administration, and can be utilized as an outline of the item, generation innovation, and so forth in any part of the industry, including those branches that don't have direct contact with 3D structure or exercises whose items or administrations are of "elusive" nature.

There has been a few learning on realistic, verbalized, 3D models of the animal body. Taking in of model from a little arrangement of 3D outputs of toy dolls in subjective postures has been done through utilizing a novel part-based shape model for figuring an underlying enrollment to the sweeps, subsequent to normalizing their posture, getting a measurable shape model, and by refining the enlistments and the model together, they precisely adjusted creature checks from various quadruped families with altogether different shapes and presents, and with the enlistment to a typical format, a shape space speaking to creatures of various species were found out (Zuffi, Kanazawa, Jacobs, \& Black, 2017).

The other research demonstrated the guideline of a programmed change from a 2D site page into a 3D site and explained about 3D portrayal strategies and change systems connected to change over a conventional two-dimensional website page into a completely utilitarian three-dimensional stage through the strategies and ideas dependent on advances like HTML, CSS, JavaScript (Hristov, \& Petkov, 2016). 
A strategy for displaying a practical inside of a structure dependent on polygonal information from stereoscopic picture sets is introduced. Subsequent to accepting the information from a past stage in a pipeline, for rendering and sending out a model into the game motor Irrlicht, 3D demonstrating project Blender is utilized. To transport the information from crude data into .obj document, shipper and vertex consolidating tools are made. .obj records are utilized for the presentation of a work in Blender and after that are traded into COLLADA group for rendering in Irrlicht (Ribe, Killian, \& Anderson, 2012).

Presentation of 3D information procurement gear and displaying strategies were given and the characters and improvements of the laser filtering framework and ImageBased Modeling and Rendering (IBMR) were likewise examined, also introduces applications of 3D modeling including tissue Engineering and heritage protection (Luan, Xie, Ying, \& Wu, 2008). An article introduced a product module intended for proficient and advantageous representation of 3D models inside the internet browser condition written in JavaScript by exploiting the HTML 5 standard, concentrating on the cell phones, unique consideration was given on effectiveness and low system utilization, likewise a proposed arrangement dependent on dynamic work spilling was contrasted and the server-side rendering approach (Sawicki \& Chaber, 2013).

The presentation of the compelling perception of 3D lattices dependent on the inherent highlights of an internet browser supporting HTML5 (Canvas, WebGL) norms was introduced and the calculations identified with dynamic work spilling over Internet system were examined and was exhibited that utilizing depicted programming, 3D models could be effectively imagined on any advanced, cell phone (web tablets, PDAs or netbooks) (Sawicki \& Chaber, 2012). A theory was exhibited to make a water reenactment utilizing WebGL and Three.js, which they talked about, will rely upon an illustrations part called a shader that produces moving water and actualizes a water surface. They expected the last recreation should look practical and be execution situated so it tends to be utilized in enormous scenes (Pereira, 2013). The examination was exhibited on the utilization of $3 \mathrm{D}$ demonstrating thought about when developing and breaking down point of view projections and shadows for the formation of a photograph sensible picture. 
The point of view of the development venture and the portrayal of its picture were given for instance. They considered the advancement of a dynamic square as a strategy for graphical information amassing and robotization of geometric improvements. The case of the dynamic square development at making a support hub was illustrated. The developments were considered as connected to Auto-CAD programming (Kheyfets, \& Vasilieva, 2017). A paper was displayed for general strategies and rules to successfully assemble a basic model made of shortcomings and skylines from normal inadequate information. A run of the mill 3D auxiliary displaying work process dependent on triangulated surfaces were portrayed. Their aspiration was not to supplant programming client directs but rather to give key ideas, standards, and methods to be connected during geomodeling undertakings, with a spotlight on quality control (Caumon, Collon-Drouaillet, Le Carlier de Veslud, Viseur, \& Sausse, 2009). A paper was composed on the primary 3D stereo motion picture made with Blender as per high philological guidelines and identified with the historical backdrop of a whole city with four distinctive geo-referenced authentic advanced territory models and a few situations, from the Etruscan up to the present day. The way of thinking made for this affirmation concentrated on open source and interdisciplinary structure (Ponti, 2013).

Drawing on their work with the Digital Life Project, they depicted their procedure for utilizing open-source programming to reproduce living creatures in $3 \mathrm{D}$, from photograph catch to movement. They analyzed the training and specialty of creating 3D models of live creatures utilizing photogrammetry (deriving estimations in 3D space utilizing photos), Focusing especially on the specialized work process utilizing open-source programming. A paper went for loosening up the demonstrating cost of the genuine space and the getting to cost on WWW proposed a straightforward methodology by utilizing beautiful pictures taken by a computerized camera or a camcorder without mutilating them and by amplifying or contracting a symbol picture and gluing it on a foundation grand picture as per the client's contribution, there had the option to imagine the profundity of the scene as pseudo-3D space. The technique was known as the picture based non-rendering (IBNR), they accentuated 
on two Focuses, the scene independency, and the stage independency (Tsukamoto, 1999).

A research paper gave knowledge into HTML 5 components, (for example, canvas and foundation specialists), JavaScript and Web Graphics Library (WebGL) advancements, and portrayed the experience picked up in utilizing the most recent developments for customer side programming by utilizing the program as an application preparing unit to empower module free running of a high caliber and processing serious game application with 3D illustrations rendering and material science impacts (Nazarov \& Galletly, 2013).

An examination inspected the impact of site dimensionality on psychological ingestion, saw usability, and, in a roundabout way, saw site value and expectations to purchase web-based utilizing the site. Their discoveries demonstrated that shopping sites utilizing 3D conditions are related without lifting a finger of utilization and lower intellectual retention, contrasted with conventional 2D sites. They believed the impact of site dimensionality on subjective assimilation is directed by client experience (Visinescu, Sidorova, Jones, \& Prybutok, 2015). A 3D reference book site of the protein nucleic corrosive and other biomolecule structures was made as a method for imparting biomolecule structures to an assorted logical group of spectators it presents basic explanation in a natural intelligent arrangement which enables individuals from mainstream researchers to contribute their very own comments. they gave a status report on their webpage by depicting propels in the web asset concentrating on highlights of potential direct use to established researchers and furthermore talked about its encouraging as a community-oriented 3d-reference book of structures just as its utilization as a supplement to logical distributions and PowerPoint introductions and portrayed the site's utilization for 3D representation in structure-related teaching method (Prilusky, 2011).

Further paper is divided into these sections, in second our overall research approach is defined, while in $3^{\text {rd }}$ section results are discussed according with modeling phase, in $4^{\text {th }}$ section we gave our hypothesis in the shape of future work and in the last section we have concluded our research in conclusion section. 


\section{RESEARCH METHODOLOGY}

The accomplishment of our undertaking and our possible strategy of making the 3D models of creatures through an open-source program and sending it into a site relies on the immense measure of point by point examine on creatures and 3D demonstrating. We have analyzed a few types of creatures and noticed the subtleties of their body parts from head to toe. Our examination centers on giving data about creatures by utilizing the 3D demonstrating system we have profited by a $3 \mathrm{D}$ displaying programming framework considered Blender and utilized it to alter a $2 \mathrm{D}$ picture into a 3D model to give it a reasonable look and feel.

\subsection{D MODEL CREATION}

The background image has been chosen for the desired 3D model and image axis was set to left, right, top, bottom, front and back through the software blender 3D. The whole body of the desired shape is created with the help of tool cube, as this tool helps in resizing, scaling and extending the shape, minor details are given through vertices as shown in Figure 1. The cube is mutilated according to the need of the shape through 'CONTROL+R'. The key ' $\mathrm{B}$ ' is used to select a specific area on the model. 'CONTROL' key was used for the selection of different points/vertices at the same time and the key ' $\mathrm{E}$ ' is used for extending the selected area for scaling of the cube the key ' $\mathrm{S}$ ' and rotation the key ' $\mathrm{R}$ ' is used. The desired shape is created with the help of tool cube.

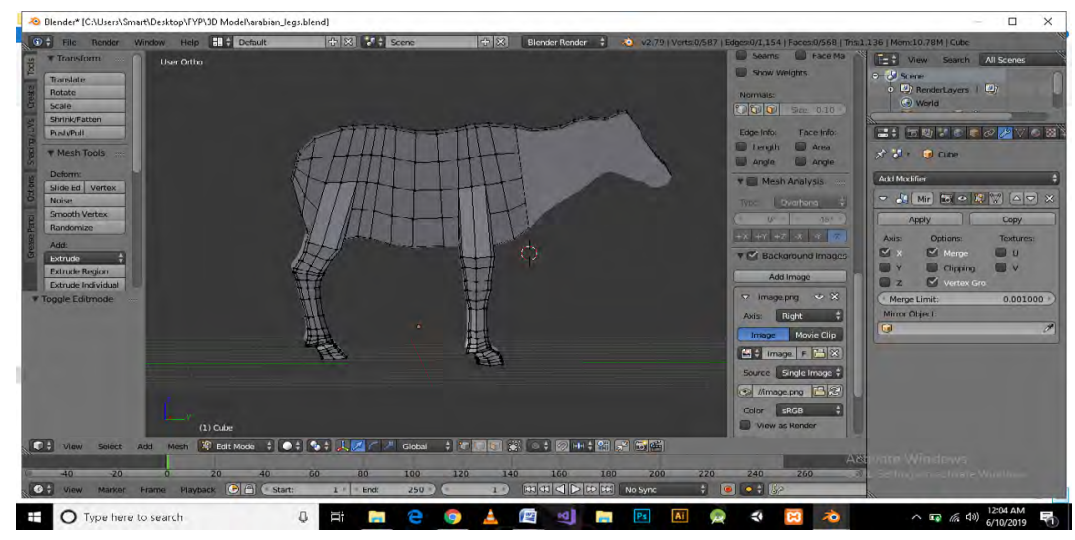

Figure 1. Mutilating the cube according to the shape of background image. 
The tools used in this process are. Cube, UV sphere and Plane and for transformation rotate, scale and translate are used for smoothing the vertices, the mesh tools are used along with shading. The 'TAB' key is used to select the working done on the object as shown in Figure 2.1 while the 'A' key selects and deselect the whole object. There is another key known as 'ESG' key it always cancels blender functions without changes and fortunately we did not use it. And for the wireframe ' $\mathrm{Z}$ ' key is used in Figure 2.2. As shown in Figure 3, to check the result of our working on the model we took the help of SHIFT $+Z$ key it showed us the rendered model.

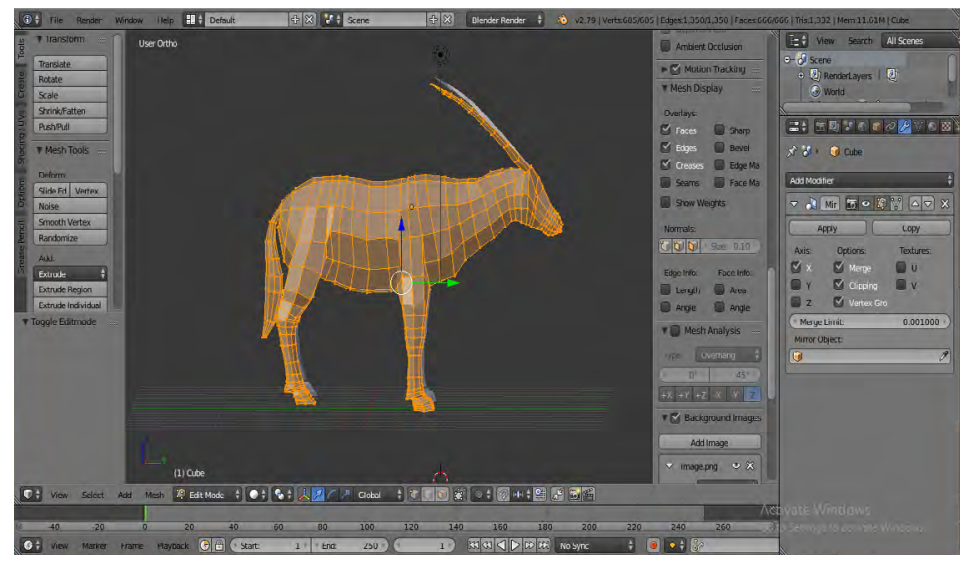

Figure 2.1. All the working done on object is selected.

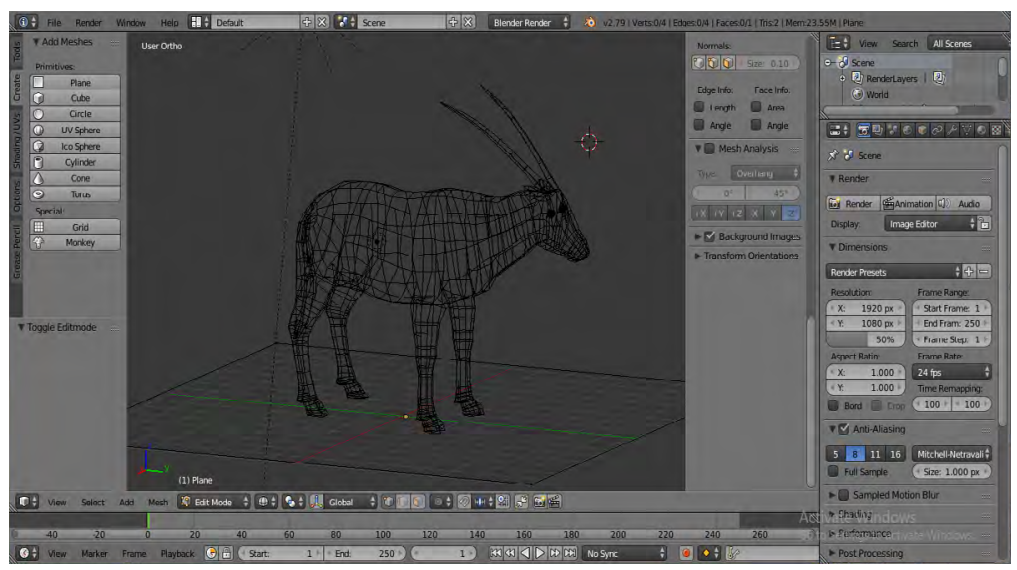

Figure 2.2. Visualizing the model in wireframe mode. 


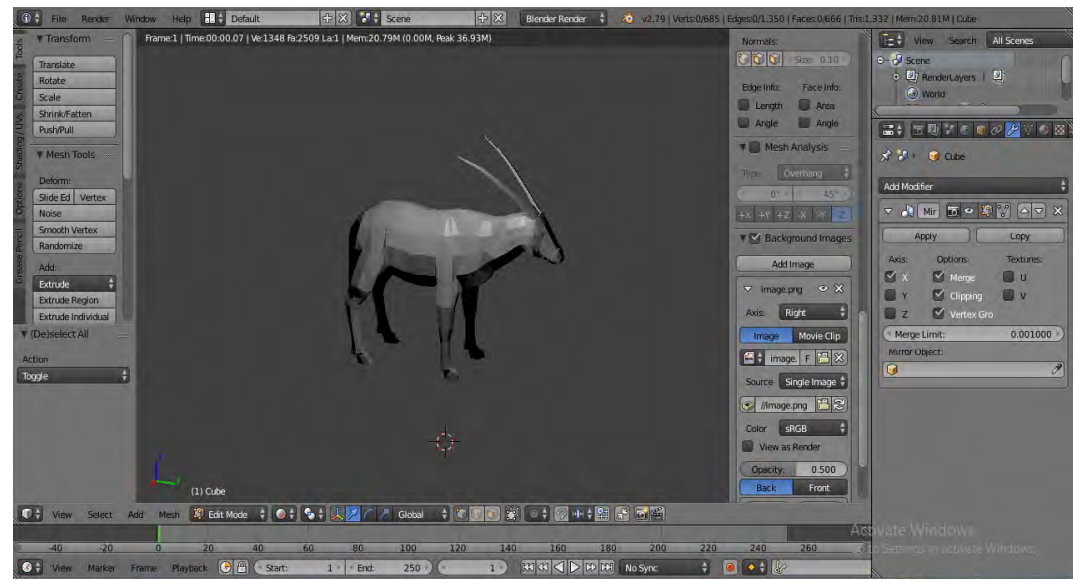

Figure 3. 3D demonstration of the object after being rendered.

\subsection{APPLYING 2D IMAGE TEXTURE USING UV WRAP}

We also used the mirror modifier to cut the mesh/cube in half. Eyes of the 3D model are made with UV sphere tool by using the key 'I' to do the inset. We used it on the face behind the eye and behind the nails. In the end after completing the shape of the 3D model we applied the texture in Figure 4, by clicking on the material tab and adding a new material on the texture tab. After selecting the image type we click on open button and selected the texture which we wanted to apply. After changing the coordinates to UV, we have changed the default window to UV editing window. Two screens appeared on our laptop.

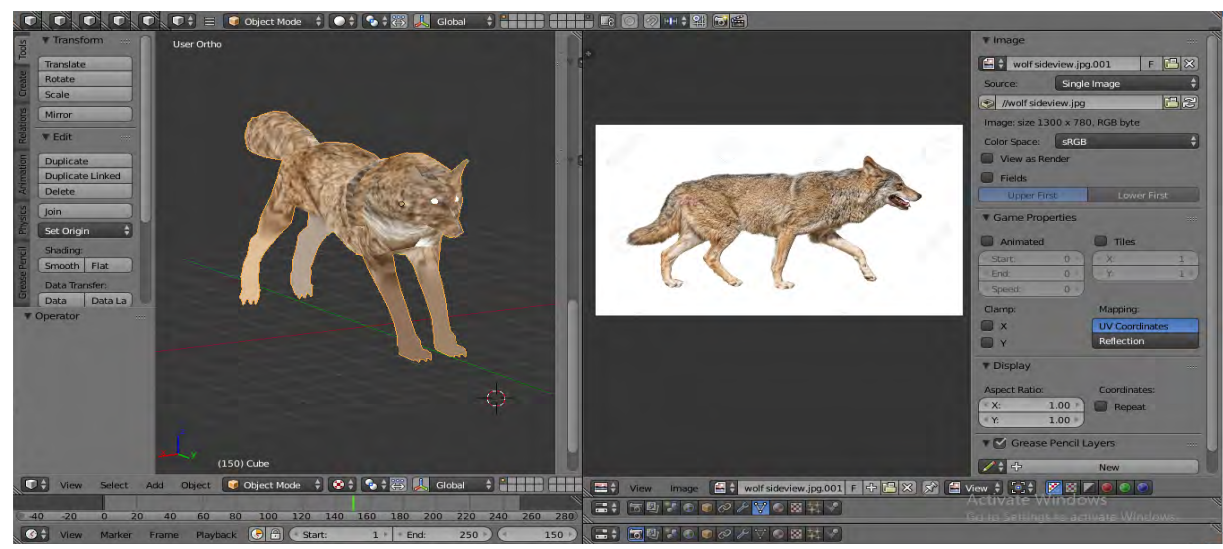

Figure 4. 2D image texture applied on object using UV wrap. 


\subsection{UNWRAPPING THE MODEL}

In UV editing window we select the texture which we had already added in default window. We then selected the model and went to the edit mode, pressed the ' $U$ ' key to unwrap the model. In the default window we selected the texture in viewport shading and the final model can be seen in viewport shading. Now we rendered our model to see the result of our working Figure 5.
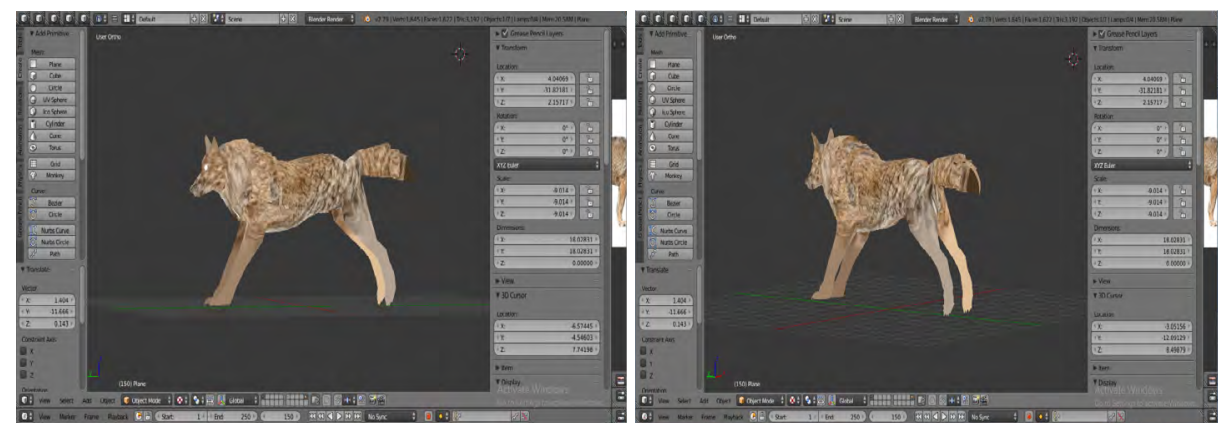

Figure 5. Rendered 3D visualization of the model after un wrapping the texture.

\section{3D MODELING AND RESULTS}

3D demonstrating is the way toward building up a numerical portrayal of any surface of an item in 3 measurements through specific programming. Blender is one of the free and open-source 3D creation software. It bolsters the sum of the 3D pipeline that is displaying, fixing, liveliness, rendering, composting, finishing, and movement following even video altering and game creation. In our project we have benefitted it from the blender 3D modeling software by using it in modifying the image into a 3D model to give it a realistic look. 3D displaying has changed the way venture plans are exhibited. It resembles consolidating various 2D illustrations and gluing them together. It has without a doubt changed the way we take a gander at undertakings and how we present data. The maxim "words usually can't do a picture justice" is really duplicated when a picture can be moved, turned, controlled, and hued. 3D demonstrating can give you a lovely one-shot image of your structure. It is progressively reasonable, speedier and simpler than 2D. You can likewise put your undertaking in other 3D models to check clearances, address access and departure, 
and even envision sightlines. Simple on the spot changes can be made to decide their reasonability.

After following all the steps above, we get a perfectly shaped textured 3D model of animal, which can benefit any individual throughout the world who is eager to learn more about animals and wildlife. The figures [6-8] are representing the rendered 3D models of animals as an example.
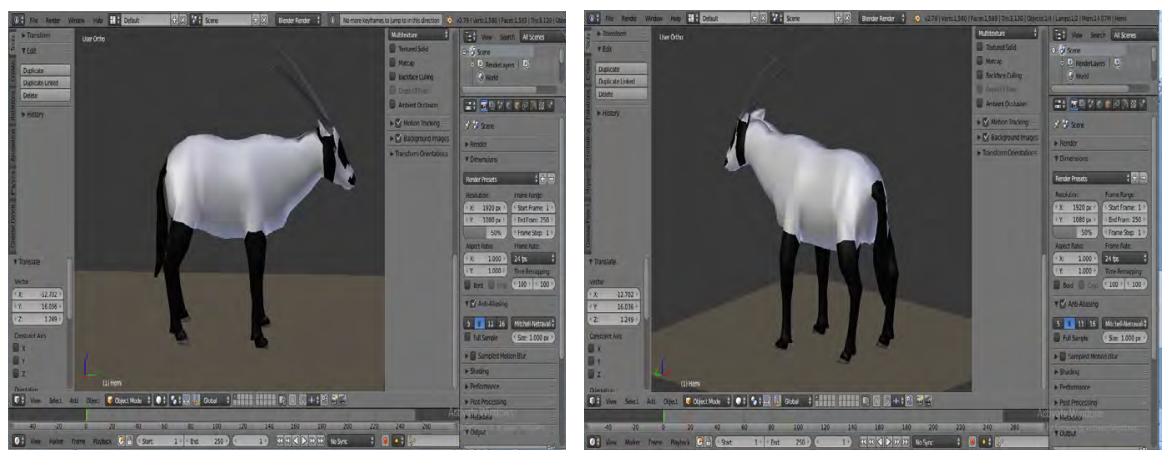

Figure 6. Rendered 3D model after applying diffuse colors in the areas.
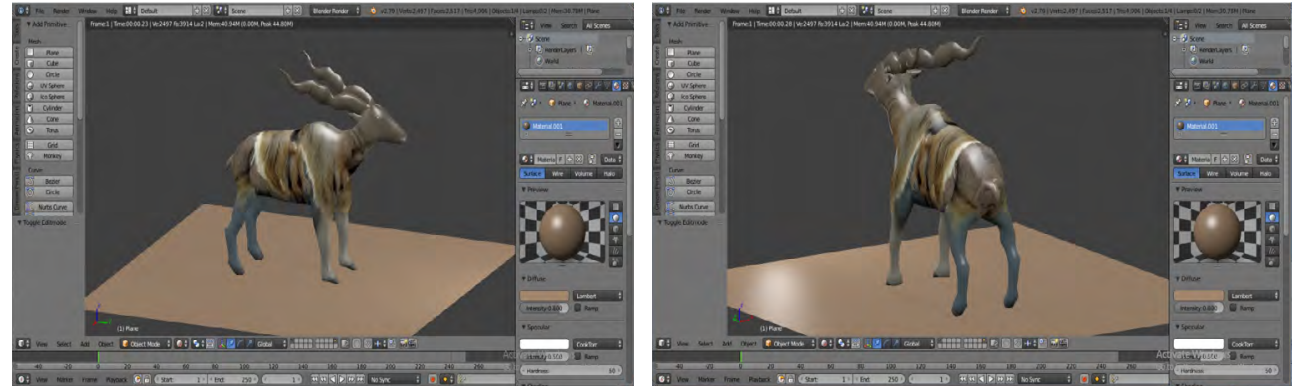

Figure 7. Rendered 3D model of the animal after unwrapping a 2D image texture.

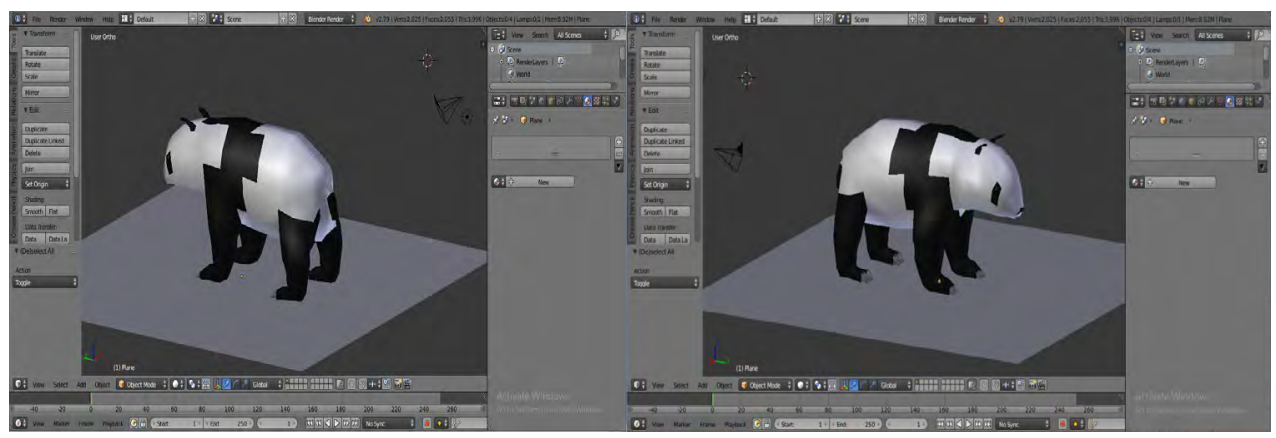

Figure 8. Rendered 3D model of a Panda after applying diffuse colors in the areas of the object. 


\section{FUTURE WORK}

In future we will deploy our 3D models to our website where users will be able rotate the model in $360^{\circ}$ and will get enough information by hovering the mouse into that body part. We will be labeling the information on models through different programming frameworks like three.js. We will accomplish our goal of making things easy by giving much information with details about wildlife. This project will serve students, families, scholars and educators around the world. This project evolves 3D multimedia educational brand to suit the constant needs of learners today.

\section{CONCLUSION}

$3 \mathrm{D}$ processing is an emerging and growing need in the multimedia domain. The successful method to create $3 \mathrm{D}$ recreation of pictures is altering three-dimensional meshes to fit the state of the item. If we want to see the object in its natural posture, it is very important to understand how the object can be eloquent and de-shaped. This research paper presents a method of deforming and articulating an object from 2D image to a 3D model. We have worked on cubes and UV sphere and by applying texture, our 3D models show that using the tools effectively works well. We hope this work of our will motivate further developments in the field of 3D blender software and it will enable completely mechanized 3D displaying of creatures from 2D pictures soon.

\section{REFERENCES}

Gaumon, G., Collon-Drouaillet, P., Le Carlier de Veslud, G., Viseur, S. \& Sausse, J. (2009). Surface-Based 3d Modeling of Geological Structures. Mathematical Geosciences, 41, 927-945. doi: https://doi.org/10.1007/s11004-0099244-2 
Hristov, P. \& Petkov, E. (2016). Development of web-based information system for visualization of three-dimensional models of museum exhibits. Conference on Digital Presentation and Preservation of Cultural and Scientific Heritage (DiPP2016). Regional Museum of History and P.R. Slaveykov Regional Public Library in Veliko Tarnovo, Bulgaria.

Kheyfets, A. L., \& Vasilieva, V. N. (2017, November). 3D Modeling as Method for Construction and Analysis of Graphic Objects. In Materials Science and Engineering Conference Series (Vol. 262, N. ${ }^{\circ}$ 1, 12104).

Luan, X., Xie, Y., Ying, L., \& Wu, L. (2008). Research and Development of 3D Modeling. International Journal of Computer Science and Network Security (IFCSNSS), 8(1), 49-53. Retrieved from: http://paper.ijcsns.org/07_book/200801/20080108.pdf

Nazarov, R., \& Galletly, J. (2013). Native browser support for 3D rendering and physics using WebGL, HTML5 and Javascript. BCI, 1036, 21-24. Retrieved from: https://www.semanticscholar.org/paper/Native-browser-support-for-3Drendering-and-physics-Nazarov-Galletly/ 1f8e52394a4d4d9de9da1 10c036ccace 5 bb97221

Pereira, K. J. (2013). Water Simulation on WebGL and Three. js. Retrieved from https://aquila.usm.edu/cgi/viewcontent. cgi? referer $=\&$ httpsredir $=1 \&$ article $=1151 \&$ context $=$ honors_theses

Ponti, F. D., Guidazzoli, A., Imboden, S., \& Liguori, M. G. (2013). A Blender open pipeline for a 3D animated historical short film. In $12^{\text {th }}$ International Symposium on Virtual Reality, Archaeology and Intelligent Cultural Heritage. doi: https:// doi.org/10.2312/PE/VAST/VAST11S/081-084

Prilusky, J., Hodis, E., Canner, D., Decatur, W. A., Oberholser, K., Martz, E., ... \& Sussman, J. L. (2011). Proteopedia: a status report on the collaborative, 3D web-encyclopedia of proteins and other biomolecules. Fournal of structural biology, 175(2), 244-252. doi: https://doi.org/10.1016/j.jsb.2011.04.011 
Ribe, J., Killian, A., \& Anderson, D. (2012). 3D Modelling in Blender Based on Polygonal Data. Midwest Instruction and Computing Symposium. Retrieved from: http://micsymposium.org/mics2012/submissions/mics2012_submission_9.pdf

Sawicki, B., \& Chaber, B. (2012). 3D mesh viewer using HTML5 technology. Przeglad Elektrotechniczny, 88(5a), 155-157.

Sawicki, B., \& Chaber, B. (2013). Efficient visualization of 3D models by web browser. Computing, 95(1), 661-673.

Tsukamoto, M. (1999, September). Image-based pseudo-3D visualization of real space on WWW. In Kyoto Workshop on Digital Cities, 288-302. Springer, Berlin, Heidelberg.

Visinescu, L. L., Sidorova, A., Jones, M. G., \& Prybutok, V. R. (2015). The influence of website dimensionality on customer experiences, perceptions and behavioral intentions: An exploration of $2 \mathrm{D}$ vs. $3 \mathrm{D}$ web design. Information $\mathcal{E}^{\circ}$ Management, 52(1), 1-17. doi: https://doi.org/10.1016/j.im.2014.10.005

Zuff, S., Kanazawa, A., Jacobs, D. W., \& Black, M. J. (2017). 3D menagerie: Modeling the 3D shape and pose of animals. In Proceedings of the IEEE Conference on Computer Vision and Pattern Recognition, 6365-6373. 
Edición Especial Special Issue Noviembre 2019

DOI: http://dx.doi.org/10.17993/3ctecno.2019.specialissue3.133-147 